\title{
ESTUDIO DE LOS FITOCONSTITUYENTES DE PLEUROTUS OSTREATUS CULTIVADO EN RESIDUOS DE PULPA DE CAFÉ
}

\section{STUDY OF THE PHYTOCONSTITUENTS OF PLEUROTUS OSTREATUS GROWN IN COFFEE PULP WASTE}

\author{
'Ángel Cuzcano-Ruiz ${ }^{\mathbb{D}}$, ${ }^{1}$ Andersson Reyes-López ${ }^{\circledR}$, ' Jessica Nieto-Juarez ${ }^{\circledR}$, 'Ingrit \\ Collantes-Díaz (1)*
}

\author{
${ }^{1}$ Grupo de Investigación en Calidad Ambiental y Bioprocesos (GICAB), Facultad de Ingeniería Química y Textil de la Universidad Nacional de \\ Ingeniería, Av. Túpac Amaru Nº 210, Rímac, Lima-Perú \\ *E-mail: ingrit_uni@hotmail.com
}

Recibido (Received): 18 /12/2019 | Aceptado (Accepted): 11/08/2020

\begin{abstract}
RESUMEN
El hongo Pleurotus ostreatus también llamado seta ostra es ampliamente cultivado por su valor nutricional, así como, por sus diferentes beneficios a la salud que se le otorgan y por su valor en remediación. El laboratorio $\mathrm{N}^{\circ} 11$ de la Universidad de Ingeniería del Perú está cultivando $P$. ostreatus haciendo uso de residuos de la pulpa de café como sustrato. El presente trabajo describe la identificación de los fitoconstituyentes mayoritarios en las fracciones apolares del extracto bruto orgánico de la seta ostra. Después de la transesterificación de una fracción rica en ácidos grasos, fue identificado el éster metílico del ácido palmítico como el mayoritario y de una segunda fracción fueron identificados los esteroides 5a,8a-epidioxi-24(R)-metilcolesta-

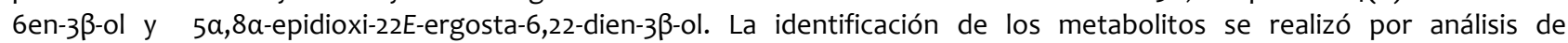
cromatografía gaseosa acoplado a espectrómetro de masas y por resonancia magnética nuclear de hidrógeno y de carbono 13.
\end{abstract}

Palabras llaves: Pleurotus ostreatus, esteroides $5 \alpha, 8 \alpha$-endoperoxidos, ácidos grasos

\section{ABSTRACT}

The mushroom Pleurotus ostreatus also called oyster mushroom is widely cultivated for its nutritional value, as well as, for its different health benefits and for its values in remediation. Laboratory $\mathrm{N}^{\circ} 11$ of the Universidad Nacional de Ingeniería of Peru is cultivating $P$. ostreatus making use of residues of coffee pulp as a substrate. The present work describes the identification of the major phytoconstituents in the apolar fractions of the organic crude extrac of oyster mushroom. After the transesterification of a fraction rich in fatty acids, the palmitic acid methyl ester was identified as the majority and of a second fraction were identified of the steroids $5 \alpha, 8 \alpha$-epidioxi-24(R)-metilcolesta- 6 en- $3 \beta$-ol and $5 \alpha, 8 \alpha$-epidioxi-22E-ergosta-6,22-dien-3 $\beta$ ol. The compounds were identified by gas chromatography analysis coupled to a mass spectrometer and by nuclear magnetic resonance of hydrogen and carbon 13.

Keywords: Pleurotus ostreatus, sterol $5 \alpha, 8 \alpha$-endoperoxides, fatty acids

\section{INTRODUCCIÓN}

Los hongos comestibles son aproximadamente 25 especies y son apreciados principalmente por sus propiedades sensoriales, tales como aroma y sabor, además de tener valor nutricional por el porcentaje de proteínas (20-50\%) y por los bajos porcentajes de carbohidratos y lípidos (<1\%), pudiendo ser utilizados en dietas con bajo porcentaje calórico siendo considerados también como fuentes de fibras, vitaminas y minerales [1]. Pleurotus ostreatus un hongo comestible de alto valor nutricional por los niveles altos de aminoácidos esenciales como arginina, alanina, glutamina y ácido glutámico; carbohidratos como trealosa, manitol, amino azúcares, etc; alto contenido en agua (> 80\%); tiene la presencia de proteínas (40\%); vitaminas como ácido ascórbico, minerales como sodio, calcio, fósforo, hierro, potasio, manganeso, cobre, zinc y los lípidos como ácido oleico, linoleico, $a$-linolénico y el ácido palmítico [2]. El presente trabajo tiene como objetivo identificar los ácidos grasos y los esteroides mayoritarios presentes en la fracción apolar del hongo $P$. ostreatus cultivados en residuos de pulpa de café, esta seta ostra fue producida por el Grupo de Investigación en Calidad Ambiental y Bioprocesos (GICAB) en la Facultad de Ingeniería Química y Textil de la Universidad Nacional de Ingeniería. La 
identificación de los compuestos se realizó por análisis de cromatografía gaseosa acoplada a espectrómetro de masas (CG-EM) y por análisis de sus espectros obtenidos por resonancia magnética nuclear de hidrógeno (RMN de ${ }^{1} \mathrm{H}$ ) y de carbono trece (RMN de $\left.{ }^{13} \mathrm{C}\right)$.

\section{ANTECEDENTES}

Pleurotus ostreatus también llamado seta ostra, es un hongo comestible, cultivado en gran escala por su alto valor nutricional en el mundo [3], lacase como antiviral [4], el péptido pleurostrina con actividad antifungal [5], el peróxido del ácido linoleico fue aislado y actuó como un excelente antinemátodos [6], Pleuran ( $\beta$-glucana) es un agente inmunomodulatoria con potencial para el tratamiento de cancer, ergotioneina, lovastatina y ácido $\gamma$-aminobutírico [7]. Un estudio del extracto acuoso de $P$. ostreatus demostró su eficiencia contra Staphylococcus aureus, Escherichia coli, Klebsiella pneumoniae, Pseudomonas aeruginosa, Streptococcus pyogenes, Shigella dysenteriae, Salmonella entérica, Candida albicans, Clavulina humicola, Trichosporon cutaneum, Aspergillus fumigatus, A. flavus, A. terreus, Diota rostrata, Coryphantha clavata y fue aislado del extracto acuoso el ácido 3-(2-aminofeniltio)-3hidroxipropanoico [8].

Dentro los metabolitos secundarios presentes en $P$. ostreatus están los flavonoides miricetina, naringenina, hespiridina, formononetina y biochanina. Dentro de los ácidos fenólicos como ácido $p$-hidroxibenzoico, ácido sinápico, ferúlico, $p$ cumárico, protocatecuico, vanílico, cafeico, gálico, homogentísico, gentísico y ácidos clorogénicos [7]; esteroides que tienen como esqueleto básico el ergosterol, presentándose con estructuras moleculares hidroxiladas y en algunos casos epidioxi, sea en las posiciones $5 \alpha, 8 \alpha$ o $5 \alpha, 9 \alpha[9,10]$.

\section{METODOLOGÍA}

\subsection{PRODUCCIÓN DE HONGOS OSTRAS PLEUROTUS OSTREATUS CULTIVADO EN RESIDUOS DE CAFÉ}

La producción de las setas ostras fue desarrollada en cuatro etapas: (1) inoculación, (2) incubación, (3) fructificación, (4) cosecha [11], como se muestra en la Fig. 1. La inoculación consistió en adicionar la semilla del hongo $P$. ostreatus en la pulpa de café (previamente pasteurizada con una humedad relativa (HR) de 70\%), a razón de $50 \mathrm{~g}$ de semilla/kg de pulpa de café, una vez inoculado el residuo de la pulpa de café, se procedió a incubar la muestra a una temperatura ( $\mathrm{T}$ ) de $27 \pm 1^{\circ} \mathrm{C}$ por un período de 20 días; seguidamente, cuando el micelio del hongo se colonizó en la pulpa de café, se procedió a fructificar las muestras a una $\mathrm{T}=18 \pm 1^{\circ} \mathrm{C}$ y HR de $90 \pm 5 \%$ por un período de 15 días, y finalmente, se procedió a cosechar las setas ostras, las cuales se utilizaron para la identificación de los metabolitos mayoritarios.

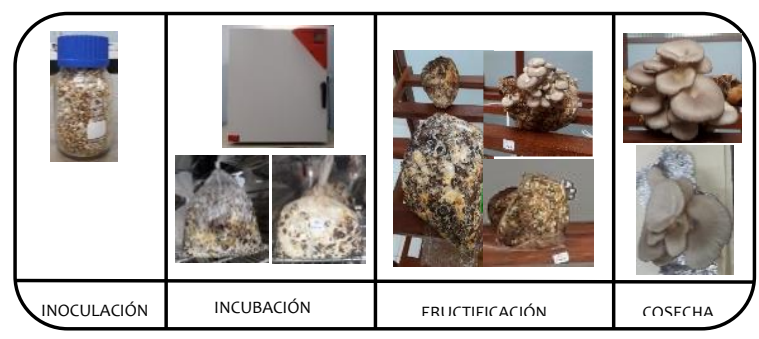

Fig. 1.- Etapas de producción del hongo Pleurotus ostreatus cultivados en residuos de pulpa de café.

\subsection{EXTRACCIÓN E IDENTIFICACIÓN DE LOS METABOLITOS SECUNDARIOS}

La muestra fresca fue pesada y congelada a $-70^{\circ} \mathrm{C}$ antes de ser liofilizada. La seta ostra liofilizada fue pulverizada en mortero y sometido a maceración en metanol por tres veces consecutivas, después de cada maceración, la muestra era filtrada y el filtrado fue secado hasta evaporación total del solvente en la campana, fue de esta manera que se obtuvo el extracto bruto orgánico (EBO) de la seta ostra.

El EBO de la muestra fue sometido a partición líquido-líquido para obtener los residuos clorofórmico $\left(\mathrm{RCHCl}_{3}\right)$, butanólico $(\mathrm{RBuOH})$ y el residuo acuoso $\left(\mathrm{RH}_{2} \mathrm{O}\right)$ según la literatura [12]. El residuo $\mathrm{RCHCl}_{3}$ de la muestra fue sometido a cromatografía en columna usando como fase estacionaria sílica gel fase normal y las fases móviles fueron éter de petróleo (EP), cloroformo $\left(\mathrm{CHCl}_{3}\right)$ y metanol (MeOH), obteniendo tres fracciones, fracción de éter de petróleo (FEP), fracción clorofórmica $\left(\mathrm{FCHCl}_{3}\right)$ y fracción metanólica (FMeOH). La FEP del residuo $\mathrm{CHCl}_{3}$ fue sometida a transesterificación para la obtención de los ésteres metílicos de los ácidos grasos según la literatura [13], para su posterior análisis por cromatografía gaseosa acoplada a espectrómetro de masas [14]. Con la intensión de identificar los esteroides mayoritarios, aproximadamente $2 \mathrm{mg}$ de la $\mathrm{FCHCl}_{3}$ del residuo clorofórmico fue sometidas a análisis por cromatografía gaseosa acoplada a espectrómetro de masas [15] y 15,9 mg de $\mathrm{FCHCl}_{3}$ fueron sometidos a análisis por RMN de ${ }^{1} \mathrm{H}$ y de $\mathrm{RMN}$ de ${ }^{13} \mathrm{C}$. 


\subsection{ANÁLISIS POR CROMATOGRAFÍA GASEOSA ACOPLADA A ESPECTRÓMETRO DE MASAS (CG-EM)}

\subsubsection{PARA LOS ÉSTERES METÍLICOS}

El análisis por cromatografía gaseosa fue realizado en un cromatógrafo a gas acoplado a un espectrómetro de masas (Shimadzu, modelo CGMSQP2010 Ultra) con las siguientes condiciones de análisis: columna capilar Restek Rtx-5MS (30 m x $0,25 \mathrm{~mm} \times 0,25 \mu \mathrm{m})$; temperatura del inyector $240^{\circ} \mathrm{C}$, temperatura del detector $230{ }^{\circ} \mathrm{C}$; impacto de electrones a $70 \mathrm{eV}$, con gas de arrastre helio a un flujo de 1,23 $\mathrm{mL} / \mathrm{min}$, con split 1/5; con un programa de temperatura de $120^{\circ} \mathrm{C}\left(2^{\prime}\right)-280^{\circ} \mathrm{C}\left(2^{\prime}\right), 2^{\circ} \mathrm{C} /$ minuto y con volumen de inyección de muestra de $3 \mu \mathrm{L}$ [14].

La identificación de las sustancias componentes fue a través de la comparación de los espectros de masas con el banco de datos NIST 62.

\subsubsection{PARA LOS ESTEROIDES}

Las condiciones de análisis de los esteroides fue: columna capilar Restek Rtx-5MS (30 m x 0,25 $\mathrm{mm} \times 0,25 \mu \mathrm{m})$; temperatura del inyector y de la interface fue de $250^{\circ} \mathrm{C}$ y $320^{\circ} \mathrm{C}$ respectivamente; impacto de electrones a $70 \mathrm{eV}$, con gas de arrastre helio a un flujo de $1,23 \mathrm{~mL} / \mathrm{min}$, con split 1/5; con un programa de temperatura de $70^{\circ} \mathrm{C}\left(5^{\prime}\right)-315^{\circ} \mathrm{C}\left(5^{\prime}\right)$, $5^{\circ} \mathrm{C} /$ minuto; y con volumen de inyección de $3 \mu \mathrm{L}$ [15].

\subsection{ANÁLISIS POR RESONANCIA MAGNÉTICA NUCLEAR}

Los espectros de resonancia magnética nuclear de ${ }^{1} \mathrm{H}$ y de ${ }^{13} \mathrm{C}$ fueron registrados en un espectrómetro Bruker de $500 \mathrm{MHz}$. Para la obtención de los espectros de hidrógeno, se trabajó a una frecuencia de $500 \mathrm{MHz}$ y para la obtención de los espectros de carbono 13 se trabajó a la frecuencia de $125 \mathrm{MHz}$. Los espectros fueron obtenidos en cloroformo deuterado $\left(\mathrm{CDCl}_{3}\right)$ de la marca MERCK.

\subsection{REACTIVOS, CONGELADORA Y LIOFILIZADOR}

Los solventes utilizados en las extracciones, partición líquido - líquido y cromatografía en columna fueron de grado para análisis de las marcas MERCK. La sílica gel para columna fue de la marca espectrum y las muestras fueron congeladas en una ultra congeladora de la marca BINDER de la serie $\mathrm{N}^{\circ}$ 20170000011259 y liofilizadas en un liofilizador de la marca LABCONCO de 2,5L de la serie 170339971 I, con bomba LABCONCO modelo 117(A65312906).

\section{ANÁLISIS DE RESULTADOS}

Fue obtenido como muestra fresca del hongo $P$. ostreatus $200,81 \mathrm{~g}$ y después de liofilizar la muestra se obtuvo $29,51 \mathrm{~g}$ de muestra seca, observándose que tiene $85,31 \%$ de agua. El porcentaje de humedad encontrado en las muestras, es un valor alto, que está conforme reporta la literatura [1]. La muestra seca fue molida en mortero y posteriormente macerada en metanol, del que se obtuvo $8,81 \mathrm{~g}$ de extracto bruto orgánico (EBO), representando el $4,42 \%$ de rendimiento. El EBO fue sometido a partición líquido-líquido, del que se obtuvo $257,9 \mathrm{mg}$ $(0,13 \%)$ de residuo clorofórmico $\left(\mathrm{RCHCl}_{3}\right), 514 \mathrm{mg}$ $(0,26 \%)$ de residuo butanólico $(\mathrm{RBuOH})$ y $6,6 \mathrm{~g}$ (3,29\%) de residuo acuoso.

El residuo clorofórmico (257,9 mg) fue sometido a cromatografía en columna abierta para la obtención de tres fracciones: fracción de éter de petróleo (FEP) del que se obtuvo 38,3 mg que representa el 43,92\%; fracción de cloroformo $\left(\mathrm{FCHCl}_{3}\right)$ del cual se obtuvo $17,9 \mathrm{mg}(20,53 \%)$ y la fracción metanólica $(\mathrm{FMeOH}) 31$ mg que representa $35,55 \%$. El total de la FEP fue sometida a transesterificación y analizado por CGEM, a través del cual fue identificado 5 ácidos grasos mayoritarios, como los presentamos en función de su porcentaje de presencia en la tabla 1 y en la Fig. 2.

TABLA I

Ácidos grasos en P. ostreatus.

\begin{tabular}{lcc}
\hline Ácidos grasos & & $\%$ \\
\hline Ácido pentadecanoico & $\mathrm{C}: 15$ & 5,63 \\
Ácido palmítico & $\mathrm{C}: 16$ & 66,54 \\
Ácido linoleico & $\mathrm{C}: 18: 2$ & 1,15 \\
Ácido oleico & $\mathrm{C}: 18: 1$ & 15,28 \\
Ácido esteárico & $\mathrm{C}: 18$ & 3,01 \\
\hline Nota: Porcentaje e identificación de los ácidos grasos mayoritarios \\
presentes en P. ostreatus
\end{tabular}

\section{Ácidos grasos y sus porcentajes}

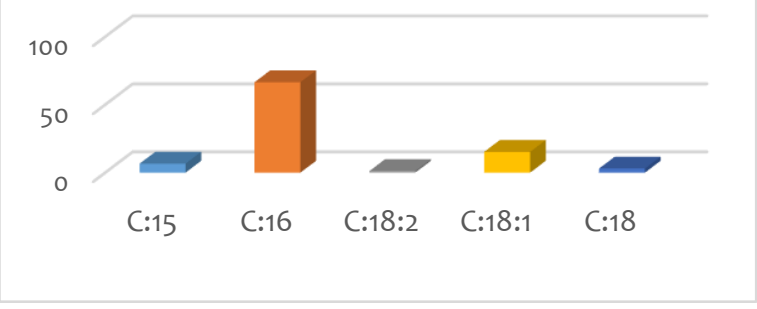

Fig. 2.- Porcentajes versus la presencia de los ácidos grasos presente en $P$. ostreatus.

De los ácidos grasos identificados en la muestra $P$. ostreatus fue observado que el ácido palmítico está presente en $66,54 \%$, valor que difiere de los 
publicado $[1,2]$ ya que lo observado en los hongos

son los ácidos oleico y linoleico los mayoritarios.

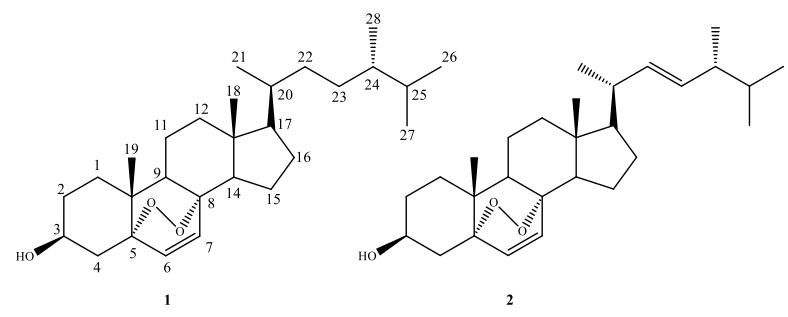

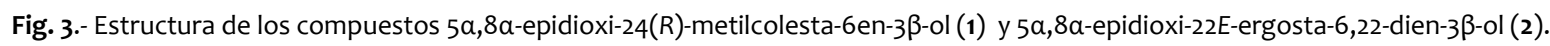
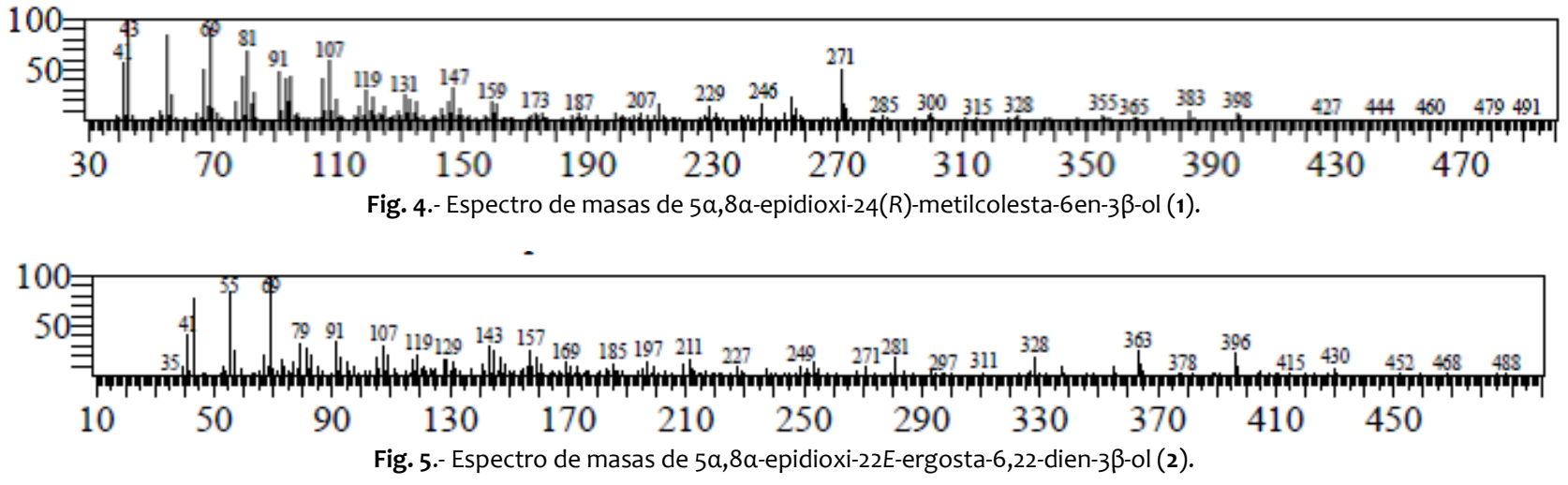

A través del análisis de los espectros de resonancia magnética nuclear y de masas obtenidos de la fracción clorofórmica $\left(\mathrm{FCHCl}_{3}\right)$ fueron identificadas las moléculas 1 y 2 (Fig. 3). Los desplazamientos químicos de RMN del compuesto 1 están de acuerdo con los datos de la literatura [16] y el espectro de masas (Fig. 4) está conforme con los datos publicados [17]. Los fragmentos observados son $\mathrm{m} / \mathrm{z}$ $398\left(\mathrm{M}^{+}-\mathrm{O}_{2}\right), 383\left(\mathrm{M}^{+}-\mathrm{O}_{2}-\mathrm{CH}_{3}\right), 365\left(\mathrm{M}^{+}-\mathrm{O}_{2}-\mathrm{H}_{2} \mathrm{O}-\right.$ $\left.\mathrm{CH}_{3}\right)$.

Compuesto 1.- Fue identificado como $5 a, 8 \alpha$-epidioxi24(R)-metilcolesta-6en-3 $\beta$-ol por análisis del espectro de $\mathrm{RMN}^{1} \mathrm{H}\left(500 \mathrm{MHz}, \mathrm{CDCl}_{3}\right) \delta 0,82(6 \mathrm{H}, \mathrm{s}, \mathrm{H}-18, d, J=$ $6,7 \mathrm{~Hz}, \mathrm{H}-27), 0,83(3 \mathrm{H}, d, J=6,7 \mathrm{~Hz}, \mathrm{H}-26), 0,88(3 \mathrm{H}$, d, J = 7,05 Hz, H-24), o,89 (3H, s, H-19), 1,00 (3H, s, H21), 3,97 (1H, $m, \mathrm{H}-3), 6,24(1 \mathrm{H}, d, J=8,55 \mathrm{~Hz}, \mathrm{H}-6)$, $6,50(1 \mathrm{H}, d, J=8,5 \mathrm{~Hz}, \mathrm{H}-7) \cdot \mathrm{RMN}^{13} \mathrm{C}\left(125 \mathrm{MHz}, \mathrm{CDCl}_{3}\right)$ $\delta$ 12,84 (C-18), 14,08 (C-28), 17,53 (C-26), 18,14 (C-21), 19,61 (C-19), 20,59 (C-11 y C-27), 23,37 (C-15), 28,79 (C16), 29,98 (C-12), 31,49 (C-2, C-23 y C-25), 33,02 (C-22), 34,66 (C-1), 35,60 (C-20), 36,79 (C-4), 36,93 (C-10), 39,32 (C-24), 44,53 (C-13), 51,06 (C-9), 51,65 (C-14), 56,17 (C-17), 66,48 (C-3), 79,39 (C-8), 82,13 (C-5), $130,71(C-7), 135,18(C-6)[16]$.

Compuesto 2.- Fue identificado como $5 a, 8 \alpha$-epidioxi22E-ergosta-6,22-dien-3 $\beta$-ol por análisis de los espectros de masas y RMN' $\mathrm{H}\left(500 \mathrm{MHz}, \mathrm{CDCl}_{3}\right) \delta$ $0,82(6 \mathrm{H}, \mathrm{s}, \mathrm{H}-18$ y $d, J=6,7 \mathrm{~Hz}, \mathrm{H}-27), 0,83(3 \mathrm{H}, d, J=$ $6,7 \mathrm{~Hz}, \mathrm{H}-26), 0,89(3 \mathrm{H}, \mathrm{s}, \mathrm{H}-19), 0,9(3 \mathrm{H}, \mathrm{d}, \mathrm{J}=6,7 \mathrm{~Hz}$, $\mathrm{H}-28), 1,00(3 \mathrm{H}, \mathrm{s}, \mathrm{H}-21), 1,22(1 \mathrm{H}, \mathrm{m}, \mathrm{H}-17), 1,23(2 \mathrm{H}, \mathrm{m}$, $\mathrm{H}-11 \mathrm{a}$ y $\mathrm{H}-12 \mathrm{a}), 1,35(1 \mathrm{H}, \mathrm{m}, \mathrm{H}-16 \mathrm{a}), 1,41(1 \mathrm{H}, \mathrm{m}, \mathrm{H}-15 \mathrm{a})$, $1,47(1 \mathrm{H}, \mathrm{m}, \mathrm{H}-25), 1,5(1 \mathrm{H}, \mathrm{m}, \mathrm{H}-9), 1,53$ (1H, $m, \mathrm{H}-11 \mathrm{~b})$, 1,56 (1H, m, H-14), 1,58 (1H, m, H-2a), 1,6 (1H, m, H15b), 1,63 (1H, m, H-1a), 1,65 (1H, m, H-16b), 1,85 (2H, $m, \mathrm{H}-2 \mathrm{~b}$ y H-24), 1,93 (1H, $m, \mathrm{H}-4 \mathrm{a}), 1,95$ (1H, $m, \mathrm{H}-1 \mathrm{~b})$, 1,96 (1H, m, H-12b), 2,01 (12, m, H-4b y H-20), 3,97 (1H, $m, \mathrm{H}-3), 6,24(1 \mathrm{H}, d, J=8,55 \mathrm{~Hz}, \mathrm{H}-6), 6,50(1 \mathrm{H}, d, J=$ $8,5 \mathrm{~Hz}, \mathrm{H}-7), 5,15(1 \mathrm{H}, d d, J=15,25 ; 8,25 \mathrm{~Hz}, \mathrm{H}-22), 5,22$ $(1 \mathrm{H}, d d, J=15,25 ; 7,6 \mathrm{~Hz}, \mathrm{H}-23) . \mathrm{RMN}^{13} \mathrm{C}(125 \mathrm{MHz}$, $\left.\mathrm{CDCl}_{3}\right) \delta 12,84$ (C-18), 17,53 (C-28), 19,61 (C-19 y C-26), 19,91 (C-27), 20,59 (C-11), 20,85 (C-21), 23,37 (C-15), 28,79 (C-16), 29,98 (C-12), 34,0 (C-25), 34,66 (C-2), 36,79 (C-4), 36,93 (C-10), 39,32 (C-1), 39,71 (C-20), 42,63 (C-24), 44,53 (C-13), 51,06 (C-9), 56,17 (C-17), 51,65 (C-14), 66,48 (C-3), 79,39 (C-8), 82,13 (C-5), 135,18 (C-6), 130,71 (C-7), 132,27 (C-23), 135,38 (C-22) [18]. El análisis del espectro de masas (Fig. 5) está conforme la referencia bibliográfica [17] y los fragmentos observados son $\mathrm{m} / \mathrm{z} 396\left(\mathrm{M}^{+}-\mathrm{O}_{2}\right), 363$ $\left(\mathrm{M}^{+}-\mathrm{H}_{2} \mathrm{O}-\mathrm{O}_{2}-\mathrm{CH}_{3}\right), 337\left(\mathrm{M}^{+}-\mathrm{O}_{2}-\mathrm{C}_{3} \mathrm{H}_{7} \mathrm{O}\right)$.

\section{CONCLUSIONES}

A través del estudio fitoquímico de las fracciones apolares de la seta ostra Pleurotus ostreatus fue 
posible identificar la presencia del ácido palmítico como compuesto mayoritario, el cual es un ácido que está ampliamente distribuido en la naturaleza. Ocurre prácticamente en todas las plantas, animales terrestres y acuáticos y es considerado como un precursor de los ácidos grasos naturales saturados e insaturados de cadenas más largas [19]. También fueron identificados dos esteroides 5a,8a-epidioxi, ambos esteroides ya aislados e identificados en hongos, siendo 5a,8a-epidioxi-22E-ergosta-6,22-dien$3 \beta$-ol la molécula más estudiada por su diversa actividad biológica como actividad antioxidante, inmunomodulatoria, antimicrobial, antitumoral, inhibidor de hemólisis, antiinflamatorio, etc y el compuesto $5 a, 8 a$-epidioxi-24(R)-metilcolesta-6en$3 \beta$-ol induce la apoptosis de las células de leucemia HL-60 [20].

\section{AGRADECIMIENTOS}

Los autores agradecen al Instituto de Investigación de la Facultad de Ingeniería Química y Textil (II-FIQT) de la Universidad Nacional de Ingeniería (UNI) por el apoyo económico brindado para la realización de este trabajo de investigación (Proyecto de Investigación Formativa 2018 código: CPDI1-2018-1) y al Proyecto de Investigación Formativa, PIF-FIQT-32017, financiado por el Vicerrectorado de Investigación (VRI-UNI), por la producción de los hongos Pleurotus ostreatus.

\section{REFERENCIAS}

[1] L. Barros, P. Baptista, D. M. Correia, S. Casal, B. Oliveira and I. C. F. R. Ferreira, "Fatty acid and sugar compositions and nutritional value of five edible mushrooms from Northeast Portugal", Food Chemistry, vol. 105, no. 1, pp. 140-145, 2007

[2] E. M. Mohamed y F. A. Farghaly, "Bioactive Compounds of fresh and dried Pleurotus ostreatus Mushroom", International Journal of Biotechnology for Wellness Industries, vol. 3, no. 1, pp. 314,2014

[3] R. C. G. Corrêa, T. Brignari, A. Bracht, R. M. Peralta and I. C. F. R. Ferreira, "Biotechnological, nutritional and therapeutic uses of Pleurotus spp. (Oyster mushroom) related with its chemical composition: A review on the past decade findings", Trends in Food Science \& Technology, vol. 50, pp. 103-117, 2016

[4] H.E. El Fakharany, B.M. Haroun, T.B. Ng and El-R. M. Redwan, "Oyster mushroom laccase inhibits hepatitis $C$ virus entry into peripheral blood cells and hepatoma cells". Protein \& Peptide Letter, vol. 17, no. 8, pp. 1031-1039, 2010

[5] K.T. Chu, L.X. Xia and T.B. Ng, "Pleurostrin, an antifungal peptide from the oyster mushroom”, Peptide, vol. 26, no. 11, pp. 2098-2103, 2005

[6] T. Satou, K. Kaneko, W. Li and K. Koike, "The toxin produced by Pleurotus ostreatus reduces the head size of nematodes", Biological and Pharmaceutical Bulletin, vol. 31, no. 4, pp. 574-576, 2008

[7] K. Piska, K. Sułkowska-Ziaja and B. Muszyńska, “Edible mushroom Pleurotus ostreatus (oyster mushroom)- its dietary significance and biological activity". Acta Scientiarum Polonorum Hortorum Cultus, vol. 16, no. 1, pp. 151-161, 2017
[8] A. M. Younis, F-S. Wu and H.H. El Shikh, "Antimicrobial activity of extracts of the oyster culinary medicinal mushroom Pleurotus ostreatus (higher Basidiomycetes) and identification of a new antimicrobial compound". International Journal of Medicinal Mushrooms, vol. 17, no. 6, pp. 579-590, 2015

[9] Y. Yaoita, K. Amemiya, H. Ohnuma, K. Furumura, A. Masaki, T. Matsuki and M. Kikichi, "Sterol constituents from five edible Mushrooms", Chemical and Pharmaceutical Bulletin, vol. 46, no. 6, pp. 944-950, 1998

[10] V. Chobot, L. Opletal, L. Jahodár̆, A. V. Patel, C. G. Dacke y G. Blunden, "Ergosta-4,6,8,22-tetraen-3-one from the edible fungus Pleurotus ostreatus (Oyster fungus)", Phytochemistry, vol. 45, no. 8, pp. 1669-1671, 1997

[11] N. Rodríguez y C. Jaramillo, "Cultivo de hongos comestibles del género Pleurotus sobre residuos agrícolas de la zona cafetera", Federación Nacional de Cafeteros de Colombia (CENICAFE), boletín técnico 27, oct. 2004.

[12] D.F. Gusmão, D.M. Estork, M.L.B. Paciencia, I.E.C. Díaz, S.A. Frana, I.B. Suffredini, A.D. Varella, R.N. Younes, L.F.L. Reis, E.F.S. Montero, and M.M. Bernardi. "Preliminary evaluation of the acute toxicity related to Abarema auriculata to mice and investigation of cytotoxicity of isolated flavonones". PharmacologyOnline, vol. 1, pp. $113-127,2013$

[13] Normas analíticas do Instituto Adolf Lutz. São Paulo, SP, Brasil: IMESP, 1985.

[14] I. E. C. Díaz, E. G. Gonçalves, M. O. M. Marques y M. Yoshida, "Incidencia de ácidos grasos en especies de araceaes", Revista de la Sociedad Química del Perú, vol. 77, no. 4, pp. 275-284, 2011

[15] Radulovic NS and Đorđević ND Steroids from poison hemlock (Conium maculatum L.): a GC-MS analysis. Journal of the Serbian Chemical Society 76, no. 11, pp.1471-1483, 2011.

[16] A. Gauvin, J. Smadja, M. Aknin, R. Faure and E-M. Gaydo, "Isolation of bioactive $5 a, 8 a$-epidioxy sterols from the marine sponge Luffariella cf. variabilis", Canadian Journal of Chemistry, vol. 78, no. 7, pp. $986-992,2000$

[17] A. A. L. Gunatilaka, Y. Gopichand, F. J. Schmitz and C. Djerassi "Minor and trace sterol in marine invertebrate. 26. Isolation and structure elucidation of nine new $5 a, 8 a$-epidioxy sterols from four marine organisms", The Journal of Organic Chemistry, vol. 46, no. 19, pp. $3860-3866,1981$

[18] M. A. Ponce, J. A. Ramirez, L. R. Galagovsky, E. G. Gros and R. Erra-Balsells, "A new look into the reaction between ergosterol and singlet oxygen in vitro", Photochemical \& Photobiological Sciences, vol. 1, pp. 749-756, 2002

[19] E. Moretto y R. Fett, Tecnologia de óleos e gorduras vegetais na indústria de alimentos. São Paulo, SP, Brasil: Varela Editora e livraria LTDA, 1998.

[20] M. Bu, B. B. Yang and L. Hu, "Natural bioactive sterol $5 a, 8 \alpha-$ endoperoxides as drug lead Compounds", Medicinal Chemistry, vol. 40, no. 10, pp. 709- 716, 2014

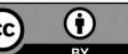

Los artículos publicados por TECNIA pueden ser compartidos a través de la licencia Creative Commons: CC BY 4.o. Permisos lejos de este alcance pueden ser consultados a través del correorevistas@uni.edu.pe 